\section{Merkel Cell Carcinoma of the Upper Eyelid: When Reconstruction Becomes a Challenge}

Luca Grassetti, Manuela Bottoni, Matteo Torresetti, Giovanni Di Benedetto

Clinica di Chirurgia Plastica e Ricostruttiva, Dipartimento di Medicina Clinica e Sperimentale, Università Politecnica delle Marche, Ospedali Riuniti di Ancona, Ancona, Italy

Correspondence: Luca Grassetti

Clinica di Chirurgia Plastica e Ricostruttiva, Dipartimento di Medicina Clinica e Sperimentale, Università Politecnica delle Marche, Ospedali Riuniti di Ancona,

Via Conca, 71-60020 Ancona, Italy

Tel: +39-071-596-3454 Fax: +39-071-596-3453

E-mail: lucagrassetti2000@gmail.com

This article was presented at the 62nd National Congress of the Italian Society of Plastic, Reconstructive and Aesthetic Surgery (SICPRE, Bari, the 25th-28th of September 2013, Italy).

No potential conflict of interest relevant to this article was reported.

Received: 10 Nov 2014 • Revised: 3 Dec 2014 • Accepted: 3 Dec 2014 pISSN: 2234-6163 • elSSN: 2234-6171

http://dx.doi.org/10.5999/aps.2015.42.2.257 • Arch Plast Surg 2015;42:257-259

Copyright @ 2015 The Korean Society of Plastic and Reconstructive Surgeons This is an Open Access article distributed under the terms of the Creative Commons Attribution Non-Commercial License (http://creativecommons.org/licenses/by-nc/3.0/ which permits unrestricted non-commercial use distribution, and reproduction in any medium, provided the original work is properly cited.

Merkel cell carcinoma (MCC) is a rare and aggressive tumor arising from Merkel cells located in the basal layer of the epidermis and in the hair follicle. MCC presents primarily in Caucasians (98.3\%) with a median age of 69 years. Ultraviolet radiation exposure and immunosuppression appear to play a role in the development of this cancer [1]. MCCs display an aggressive clinical course with a high rate of local recurrence (26\%-60\%), regional lymph node invasion (45\%-91\%), and metastasis to the lung, liver, brain, bone, and skin (18\%-52\%). Eyelid MCCs account for $5 \%-10 \%$ of all MCCs [2]. Although clinical management is often discussed, few cases involving the eyelid location have been described. For anatomical reasons and due to the rarity of the tumor, standard site-specific treatment regimens have not been defined [3]. We present a case of Merkel cell carcinoma of the eyelid that was treated with the aim of achieving surgical eradication of the tumor, as well as satisfactory aesthetic and functional outcomes.

A 75-year-old Caucasian female was referred to our clinic 1 month after presentation of a rapidly growing lesion of the right upper eyelid. Clinical examination showed a solitary, asymptomatic, $1 \mathrm{~cm}$ diameter, pink-red vascularized cutaneous nodule of the middle third of the right upper eyelid (Fig. 1). Histological examination of an incision biopsy established the diagnosis of MCC. Clinically, there was no evidence of lymph node involvement in the head, neck, or axillae. The preoperative staging of MCC included a whole-body computed tomography (CT) spiral scan with contrast and positron emission tomography $\left({ }^{18} \mathrm{~F}\right.$-fluorodeoxyglucose positron emission tomography/CT). Screening did not reveal any metastases, classifying the patient in IB stage (T1$\mathrm{cN} 0-\mathrm{M} 0$ ). Under general anesthesia, we performed a wide excision of the whole upper right eyelid containing the lesion with $1 \mathrm{~cm}$ free margins and preserving the upper part of the lid levator muscle. Intraoperative sections showed no tumor presence in the edges. A full thickness fasciocutaneous Fricke flap from the temporal region was raised, and passed subcutaneously, after de-epithelialisation, to reconstruct the upper lid (Fig. 2). The pedicle, based on peripheral branches of the superficial temporal artery, had previously been identified by ultrasonography about $1 \mathrm{~cm}$ from the lateral orbit rim. In order to preserve palpebral functionality, the internal lining (i.e., fascia) of the flap was anchored to the levator muscle.

Conjunctival reconstruction using a mucosal graft taken from the lower lip was also necessary for the internal lining. Histologic examination showed a MCC involving the skin and the conjunctiva of the upper lid positive to CK-20 and synaptophysin, with complete excision. The patient underwent adjuvant radiotherapy. At the 1 year follow-up, the patient was free from disease and continued to demonstrate a good functional and cosmetic outcome (Figs. 3, 4).

$\mathrm{MCC}$ is a rare skin cancer with a mortality rate higher than melanoma. In its early stage, the neoplasm does not demonstrate specific clinic characteristics. Given the rarity of the tumor and the possibility of misdiagnosis, a high index of suspicion is necessary, especially in immunosuppressed patients. The

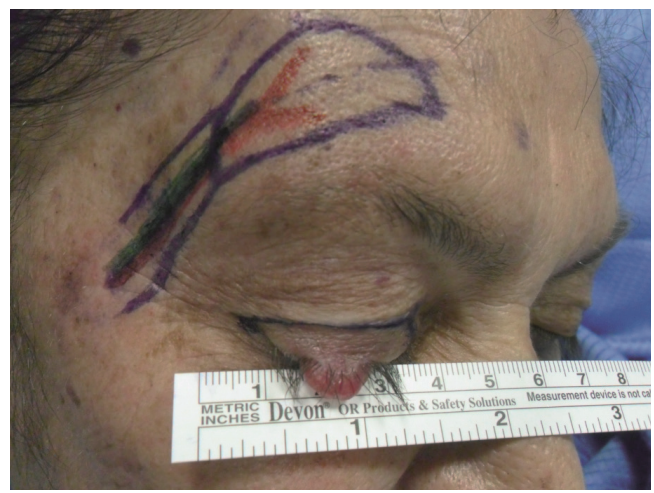

Fig. 1.

Preoperative planning of a $1 \mathrm{~cm}$ nodular merkel cell carcinoma excision and reconstruction with a Fricke flap. The vascular pedicle is marked in red after hand-held Doppler scanning. 
Fig. 2.

An intraoperative image showing the tunneled Fricke flap inset after merkel cell carcinoma excision and an oral mucosal graft positioned with direct closure of the donor site along the natural wrinkle.

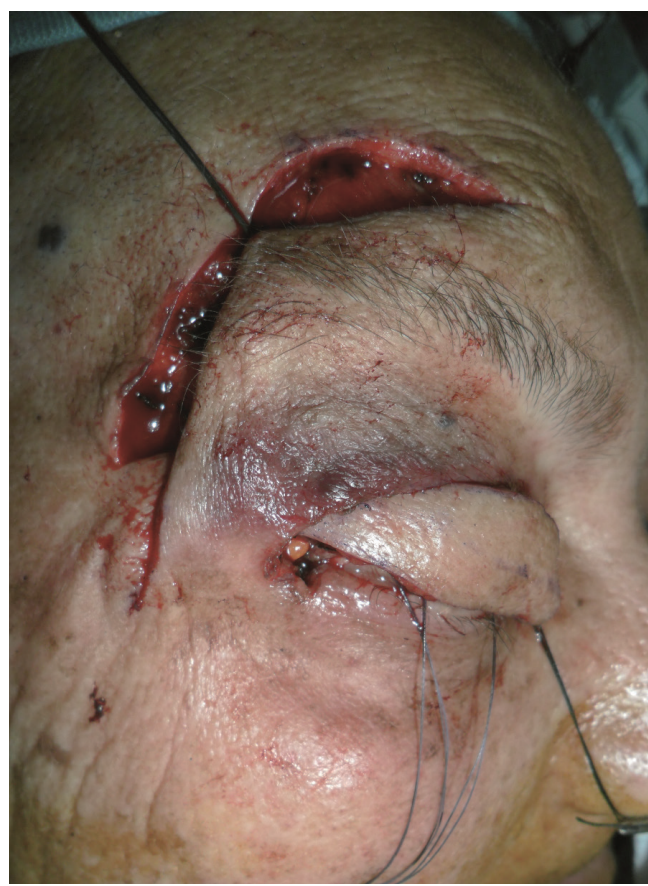

diagnosis is essentially histological. The staging of the tumor necessitates a whole-body CT spiral scan and PET. In stage I and II, wide local excision of the primary lesion with 1 to $2 \mathrm{~cm}$ margins investing fascia remains the gold standard. Mohs' micrographic surgery or modified Mohs' surgery can be considered in critical tissue sparing cases such as for facial MCC. Of course, in the lids, excision needs to involve the full thickness of the lid itself. The necessity of a sentinel lymph node is controversial, but it can be helpful in staging and for prognosis. The use of local adjuvant radiation therapy (RT) decreases the risk of recurrences. The plasmatic dosage of chromogranin $A$ and NSE can be useful in post-treatment follow-up. Chemotherapy treatment must be considered in cases of advanced disease. A number of techniques have been described for eyelid reconstruction after a full thickness excision. A Tenzel flap (or semicircular rotation flap) is useful for covering defects of $40 \%$ $60 \%$ of the eyelid, while in our case we performed a total upper lid excision [4]. The Cutler-Beard, or bridge flap, is the classic eyelid sharing procedure, but it requires prolonged occlusion of the eye and a twostage procedure (division of the flap traditionally occurs in 6 weeks), and it can produce persistent lower eyelid instability and upper lid entropion. The Mustardé lid flap utilizes a two-stage transposition of the lower lid margin including the lashes. In this case also the flap division occurs in 6 weeks. Further disadvantages include the potential irritation of the

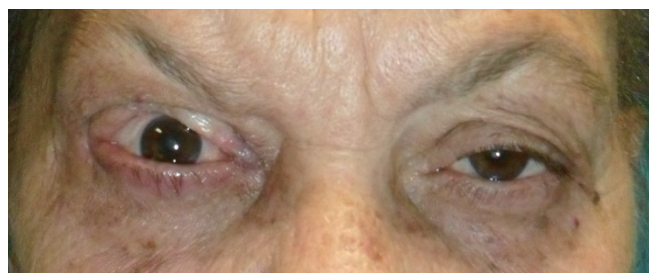

Fig. 3.

Six-month postoperative result after complete functional recovery (eyes wide open).

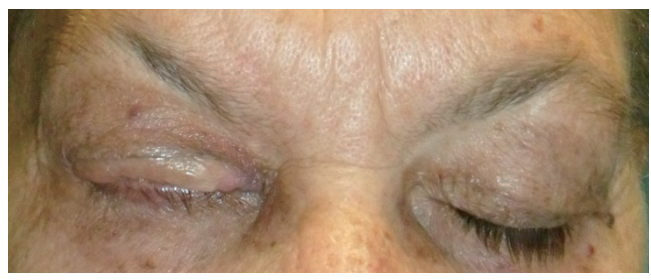

Fig. 4.

Six-month postoperative result after complete functional recovery (eyes closed).

cornea by the pedicle, the need for full-thickness lower eyelid reconstruction, and a poor cosmetic outcome of the lower lid. The Tripier flap has also been reported to be used in this situation, but it can result in entropion due to the lack of rigid support to replace the tarsus. The forehead flap is generally one of last choice, as it is a very thick flap that requires a two-stage procedure (usually 2 weeks are required for division of the flap). This flap can be combined with various other procedures to repair full-thickness defects of the eyelids [5].

The temporal forehead flap described by Fricke is a transposition flap used for full-thickness eyelid defects. This flap allows lower lid sparing, avoiding prolonged eye occlusion, with a single-step surgical procedure. The vascular pedicle is reliable, and fast and easy to raise and harvest. Usually the flap donor site scar can be easily hidden in the wrinkles or in the brow margin; besides, one step reconstruction of the lashes by using eyebrows can be performed. For these reasons, our choice has ensured long-term eye protection, good functionality, and an aesthetic shape.

Due to the rarity of this tumor, there is no consensus on its optimal treatment, especially with regard to palpebral localization. We conclude that an early and wide local excision of the primary tumor either alone or with $\mathrm{RT}$ is actually the more appropriate treatment for stage IA and IB MCC.

Composite full-thickness reconstruction is always required in MCC. For this reason, the physician who 
approaches such a case should be confident in performing this kind of flap.

\section{References}

1. Medina-Franco H, Urist MM, Fiveash J, et al. Multimodality treatment of Merkel cell carcinoma: case series and literature review of 1024 cases. Ann Surg Oncol 2001;8:204-8.

2. Bichakjian CK, Lowe L, Lao CD, et al. Merkel cell carcinoma: critical review with guidelines for multidisciplinary management. Cancer 2007;110:1-12.

3. Baccarani A, Pompei B, Pedone A, et al. Merkel cell carcinoma of the upper eyelid: presentation and management. Int J Oral Maxillofac Surg 2013;42:711-5.

4. DiFrancesco LM, Codner MA, McCord CD. Upper eyelid reconstruction. Plast Reconstr Surg 2004;114:98e-107e.

5. Stanizzi A, Grassetti L. Full-thickness lower eyelid reconstruction: an easy and reliable method of reinforcing the forehead flap with fascia lata. Plast Reconstr Surg 2012;129:376e-377e.

\section{Cutaneous T-Cell Lymphoma with Nasal Necrosis}

Katherine Shaum, Joyce Aycock

Division of Plastic and Reconstructive Surgery, University of Colorado School of Medicine, Aurora, CO, USA

Correspondence: Joyce Aycock

University of Colorado Anschutz Medical Campus, 12631 East 17th Avenue, C 309, C0 80045, Aurora, USA

Tel: +1-303-724-2792, Fax: +1-303-724-2825

E-mail: Joyce.aycock@ucdenver.edu

No potential conflict of interest relevant to this article was reported.

Received: 9 Aug 2014 • Revised: 29 Sep 2014 • Accepted: 7 Oct 2014 pISSN: 2234-6163 • elSSN: 2234-6171

http://dx.doi.org/10.5999/aps.2015.42.2.259 • Arch Plast Surg 2015;42:259-261

Copyright (C) 2015 The Korean Society of Plastic and Reconstructive Surgeons This is an Open Access article distributed under the terms of the Creative Commons Attribution Non-Commercial License (http://creativecommons.org/licenses/by-nc/3.0/) which permits unrestricted non-commercial use, distribution, and reproduction in any medium, provided the original work is properly cited.

Mycosis fungoides is a rare T-cell cutaneous lymphoma that poses a unique diagnostic challenge given its heterogeneous presentation. The refractory case presented here highlights the need for advances in early diagnostic technologies.

A 73-year-old diabetic man presented with erythema and necrosis of the nose. He initially had a $7 \mathrm{~mm}$ squamous cell carcinoma on the nose which was excised with Mohs surgery. The wound rapidly progressed to full thickness necrosis of the left nasal ala without pain or fever. During subsequent reconstruction with a nasolabial flap and cartilage graft, biopsies of the nasal margin showed severe inflammation without malignancy. The reconstructed area failed to heal and serial cultures grew multiple species of bacteria which were treated with several courses of antibiotics. Erythema of the nose and face continued to progress over 7 months and new lesions appeared, including skin necrosis at the site of previous left ala flap, right dorsum of nose and right cheek (Fig. 1). Computed tomography (CT) scan showed no involvement of sinuses or facial bones. Repeat biopsy showed acute and chronic inflammation with no malignancy and no organisms. A few weeks later a new erythematous plaque developed on the back (Fig. 2) and biopsy showed lymphocytic infiltrate with a monoclonal T-cell population (Figs. 3, 4). Repeat assay of previous nasal biopsy showed the same T-cell receptor gene rearrangement leading to the diagnosis of cutaneous T-cell lymphoma, consistent with mycosis fungoides (MF). The patient was staged with positron emission tomography-CT scan showing no visceral disease despite developing new lesions in his groin in addition to new lesions on his back and face. He was treated with a combination of systemic chemotherapy, radiation and phototherapy. At 16 month follow up he has persistent skin lesions, with progression of tumor burden.

This case highlights the diagnostic difficulty seen

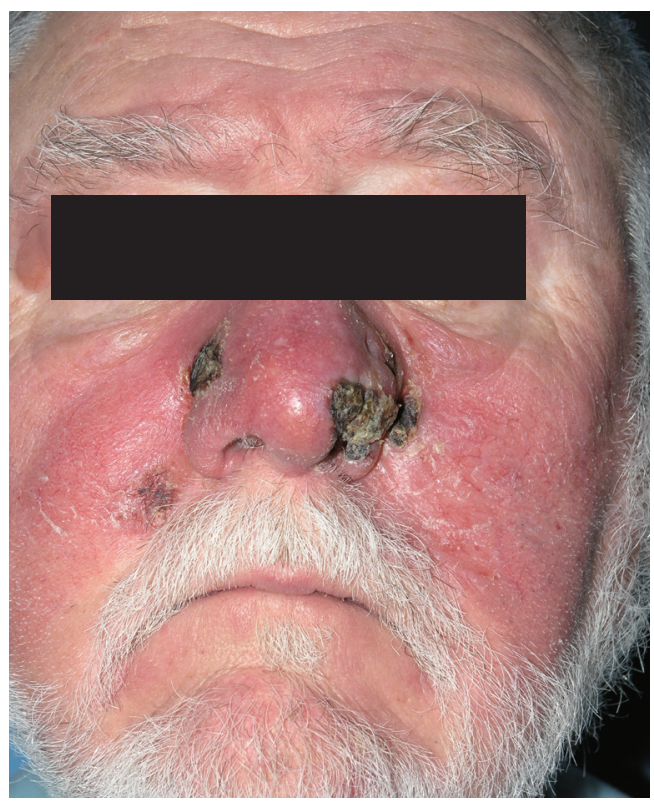

Fig. 1 ,

Facial involvement of mycosis fungoides with significant nasal necrosis at site of previous nasolabial flap. 